\title{
Phase Equilibria of Quasi-Ternary Systems Consisting of Multi-Component Polymers in a Binary Solvent Mixture VII. Co-Solvency
}

\author{
Shigenobu MATSUDA and Kenji KAMIDE* \\ Fundamental Research Laboratory of Fibers and Fiber-Forming Polymers, \\ Asahi Chemical Industry Co., Ltd., \\ 11-7 Hacchonawate, Takatsuki, Osaka 569, Japan
}

(Received May 15, 1986)

\begin{abstract}
An attempt was made to study theoretically the conditions of occurrence of cosolvency phenomena for quasi-ternary components systems consisting of a multicomponent polymer dissolved in a binary solvent mixture. For this purpose, Kamide and Matsuda theory on spinodal curve (SC) and critical solution point (CSP) for the quasi-ternary systems [Polym. J., 18, 347 (1986)] was employed. The co-solvency occurs generally only when the total polymer volume fractions $v_{\mathrm{p}}$ at two spinodal points $\left(v_{1}=0\right.$ and $\left.v_{2}=0\right), v_{\mathrm{p}}{ }^{(1)}$ and $v_{\mathrm{p}}{ }^{(2)}$ satisfy the following two inequalities concurrently: $0<v_{\mathrm{p}}{ }^{(1)}<1$ and $0<v_{\mathrm{p}}{ }^{(2)}<1$. (Here, $v_{1}$ and $v_{2}$ are the volume fractions of solvents 1 and 2.) SC, CSP, and the cloud point curve (CPC) were calculated for a wide range of three thermodynamic interaction parameters $\chi_{12}, \chi_{13}$, and $\chi_{23}$ (the suffixes 1 and 2 mean solvents 1 and 2 , respectively, the suffix 3 , the polymer). Co-solvency occurs generally in the cases when (1) original polymer has smaller weight-average degree of polymerization $X_{w}{ }^{0}$, (2) the polymer has the smaller ratio of $X_{w}{ }^{0}$ to the number-average degree of polymerization $X_{n}{ }^{0}$ (i.e., $X_{w}{ }^{0} / X_{n}{ }^{0}$ ), and (3) the initial polymer volume fraction $v_{\mathrm{p}}{ }^{0}$ is smaller than that of the critical concentration $v_{\mathrm{p}}{ }^{\mathrm{c}}$. There exists a specific $\chi_{12}$ value yielding the most wide composition range of co-solvency of the system concerned (for example $\chi_{12}=1.4$ for $X_{w}{ }^{0}=300$ ). When $\chi_{12}=0$, no co-solvency occurs. For larger $\chi_{12}$ (but not larger than 2.0), smaller $\chi_{13}$, and smaller $\chi_{23}$, co-nonsolvency was theoretically predicted to occur and in this case the system has two Flory solvent compositions.
\end{abstract}

KEY WORDS Co-Solvency / Quasi-Ternary System / Binary Solvent Mixture / Spinodal Curve / Critical Solution Point / Thermodynamic Interaction Parameters /

Even if two solvents are poor against a given polymer when they are used separately, a binary mixture consisting of these two solvents sometimes dissolves the polymer. In this sence the binary mixture acts as good solvent. This phenomenon is conventionally called as co-solvency, first discovered in 1920 's experimentally for cellulose nitrate solution sytems. ${ }^{1}$ Thereafter, co-solvency has been observed for numerous polymer/ two nonsolvents mixture systems including cellulose acetate (CA)/chloroform/ethyl alcohol, ${ }^{2}$ polystyrene (PS)/acetone/n-propyl laurate ${ }^{3} \quad \mathrm{PS} /$ methylcyclohexane $(\mathrm{MCH}) /$ acetone ${ }^{4} \quad \mathrm{PS} /$ acetone/cyclohexanol, ${ }^{5}$ PS/ $\mathrm{MCH} /$ diethyl ether, ${ }^{5}$ PS/acetone/diethyl ether, ${ }^{6,7}$ poly(methyl methacrylate) (PMMA)/ benzyl alcohol/sec-butyl chloride, ${ }^{8} \operatorname{poly}(p$ tert-butyl phenyl)methacrylate/acetone $/ \mathrm{CH},{ }^{9}$ PMMA $/ \mathrm{CCl}_{4} /$ tert-butyl chloride, ${ }^{10}$ PMMA/ $\mathrm{CCl}_{4} /$ butyl chloride. ${ }^{10,11}$

Scott $^{12}$ calculated co-existing curve for

\footnotetext{
* To whom all correspondences should be addressed.
} 
ternary system of monodisperse polymer/ binary solvent mixture, showing that under the conditions of infinite molecular weight polymer with $\chi_{12}=2.0$ and $\chi_{13}=\chi_{23}=0.75$, there exist two binodal curves (in other words, there are two two-phase separation regions).

Bamford and Tompa ${ }^{13}$ showed, using the conditional equation giving critical solution point (CSP), proposed by Scott, ${ }^{12}$ that two CSP are theoretically expected to exist for $\mathrm{CA} /$ chloroform/ethyl alcohol system. Noting chloroform as solvent 1 and ethyl alcohol as solvent 2 and assuming three thermodynamic interaction parameters between solvent 1 and solvent $2\left(\chi_{12}\right)$ solvent 1 and polymer $\left(\chi_{13}\right)$, and solvent 2 and polymer $\left(\chi_{23}\right)$ to be $1.10,0.56$ and 1.00 , respectively and the degree of polymerization of the polymer $X=953$, they calculated the volume fractions of solvent 1 , solvent 2 , and the polymer at CSP. These critical volume fractions, $v_{1}{ }^{\mathrm{c}}, v_{2}{ }^{\mathrm{c}}$ and $v_{\mathrm{x}}{ }^{\mathrm{c}}$ (c denotes CSP) were $0.912,0.057$, and 0.031 for one CSP, and $0.511,0.464$, and 0.025 for another, respectively. Note that $\chi_{23}$ parameter employed by them was not experimentally determined and Scott equations of CSP do not satisfy the thermodynamic requirement. In other words, these equations are not correct, as very recently pointed out by Kamide and Matsuda. ${ }^{14}$ Using Scott's equations of CSP (eq 26 and 27 in ref 12) for the ternary system $\left(X=953, \quad \chi_{12}=1.10, \quad \chi_{13}=0.56\right.$, and $\chi_{23}=$ $1.00)$ we obtain $\left(v_{1}{ }^{\mathrm{c}}, v_{2}{ }^{\mathrm{c}}, v_{\mathrm{x}}{ }^{\mathrm{c}}\right)=(0.985,0.0580$, $0.0335)$ and $(0.5089,0.4604,0.0307)$. By use of Tompa's equation of CSP (eq 7.27 and 7.29 in ref. 15) for the same system we obtain not only a correct $\operatorname{CSP}\left(v_{1}{ }^{\mathrm{c}}, v_{2}{ }^{\mathrm{c}}, v_{\mathrm{x}}{ }^{\mathrm{c}}\right)=(0.9110$, $0.0583,0.0307)$ and $(0.5112,0.4630,0.0258)$ but also an incorrect $\operatorname{CSP}\left(v_{1}{ }^{\mathrm{c}}, v_{2}{ }^{\mathrm{c}}, v_{\mathrm{x}}^{\mathrm{c}}\right)=$ $(0.3483,0.6494,0.00228)$. If we use KamideMatsuda's equation of CSP (eq $10^{\prime}$ and $11^{\prime}$ in ref 14 ) for the above system we obtain correct $\left(v_{1}{ }^{\mathrm{c}}, v_{2}{ }^{\mathrm{c}}, v_{\mathrm{x}}{ }^{\mathrm{c}}\right)$ only.

Tompa $^{15}$ predicted without showing detailed derivation that for infinite molecular weight polymer, co-solvency occurs if the following conditions are satisfied.

$$
\begin{aligned}
& 2\left(\chi_{12} \chi_{13}+\chi_{13} \chi_{23}+\chi_{23} \chi_{12}\right) \\
& -\left(\chi_{12}{ }^{2}+\chi_{13}{ }^{2}+\chi_{23}{ }^{2}\right)>0 \\
& 2> \\
& \chi_{12}>\chi_{13}+\chi_{23}-1 \\
& \quad+\left(2 \chi_{13}-1\right)^{1 / 2}\left(2 \chi_{23}-1\right)^{1 / 2}
\end{aligned}
$$

Tompa explained co-solvency as follows: when the cohesive energy density of the polymer lies between those of two solvents, the solvent mixture may have on average the cohesive energy density near to that of the polymer and in consequence the binary mixture of two nonsolvents dissolves the polymer. ${ }^{15}$

The second virial coefficient $A_{2}$ by the light scattering and the limiting viscosity number $[\eta]$ have been investigated in correlation to co-solvency phenomena. ${ }^{4-11,16-21} A_{2}$ and [ $\left.\eta\right]$ were shown to exhibit maximum at a specific composition of binary mixture and these were discussed in terms of selective adsorption coefficient $\lambda .^{22}$ The solubility parameter $\delta,{ }^{4,5}$ the excess free energy of mixing $\Delta G^{\mathrm{E}}{ }_{12},{ }^{4,16}$ the long range intermolecular interaction parameter $B^{10}$ in the two-parameter theory estimated by Stockmayer-Fixman plot, ${ }^{23}$ coil $\operatorname{size}^{4,5,8}$ and the linear expansion factor $\alpha^{24}$ were also studied as a function of binary mixture composition. In particular, Katime and Ochoa $^{11}$ attempted to explain co-solvency by using the solubility parameter defined by Koenhen and Smolders. ${ }^{25}$ It should be noted that the physical quantities such as $A_{2},[\eta], \lambda, \delta, \Delta G^{\mathrm{E}}{ }_{12}, B$, and $\alpha$ do not have "the relation of cause and effect" against occurrence of co-solvency, but may be indirectly correlated with the co-solvency.

This article intends to disclose the thermodynamical conditions of occurrence of cosolvency using the Kamide-Matsuda theory $^{14,26}$ of phase equilibria of polydisperse polymer/solvent $1 /$ solvent 2 (i.e., quasiternary) systems and to demonstrate theoretically that co-solvency is very generally ob- 
servable phenomena for the quasi-ternary systems.

\section{THEORETICAL BACKGROUND}

The conditions of spinodal and neutral equilibrium of the quasi-ternary system are given by eq 3 and 4 , respectively. ${ }^{14}$

$$
\begin{gathered}
\left(\frac{1}{v_{1}}+\frac{1}{v_{2}}-2 \chi_{12}\right)\left(\frac{1}{v_{\mathrm{p}} X_{w}{ }^{0}}+\frac{1}{v_{1}}-2 \chi_{13}\right) \\
-\left(\frac{1}{v_{1}}+\chi_{23}-\chi_{13}-\chi_{12}\right)^{2}=0 \\
{\left[\frac{1}{v_{\mathrm{p}} X_{w}{ }^{0}}\left(\frac{1}{v_{1}{ }^{2}}-\frac{1}{v_{2}{ }^{2}}\right)+\frac{1}{v_{1}{ }^{2}}\left(\frac{1}{v_{2}}-2 \chi_{23}\right)\right.} \\
\left.-\frac{1}{v_{2}{ }^{2}}\left(\frac{1}{v_{1}}-2 \chi_{13}\right)\right]\left(\frac{1}{v_{\mathrm{p}} X_{w}{ }^{0}}+\frac{1}{v_{1}}-2 \chi_{13}\right) \\
-\left(\frac{1}{v_{1}}+\chi_{23}-\chi_{13}-\chi_{12}\right)\left[\frac{1}{v_{1}{ }^{2}}\left(\frac{1}{v_{2}}-2 \chi_{23}\right)\right. \\
+\frac{1}{v_{1}{ }^{2} v_{\mathrm{p}} X_{w}{ }^{0}}+\frac{X_{z}{ }^{0}}{v_{\mathrm{p}} X_{w}{ }^{0}}\left\{\frac{1}{v_{1} v_{2}}-\frac{2 \chi_{13}}{v_{2}}-\frac{2 \chi_{23}}{v_{1}}\right. \\
+2\left(\chi_{12} \chi_{13}+\chi_{13} \chi_{23}+\chi_{23} \chi_{12}\right) \\
\left.\left.-\left(\chi_{12}{ }^{2}+\chi_{13}{ }^{2}+\chi_{23}{ }^{2}\right)\right\}\right]=0
\end{gathered}
$$

with

$$
v_{2}^{(2)}=1-v_{\mathrm{p}}^{(2)}
$$

At $v_{2}=0$ SC and SP are independent of $\chi_{12}$ and $\chi_{23}$ and a single function of $\chi_{13}$ in the form:

$$
v_{\mathrm{p}}+\frac{v_{1}}{X_{w}{ }^{0}}-2 \chi_{13} v_{1} v_{\mathrm{p}}=0
$$

By solving eq 8 , the composition of $\mathrm{SP}\left(v_{1}{ }^{(1)}\right.$, $\left.v_{\mathrm{p}}{ }^{(1)}\right)$ at $v_{2}=0$ are given by eq 9

$$
\begin{aligned}
v_{p}^{(1)}= & \frac{1}{2}\left[1-\frac{1}{2 \chi_{13}}+\frac{1}{2 \chi_{13} X_{w}{ }^{0}}+\left\{\left(1-\frac{1}{2 \chi_{13}}\right.\right.\right. \\
& \left.\left.\left.+\frac{1}{2 \chi_{13} X_{w}{ }^{0}}\right)^{2}-\frac{2}{\chi_{13} X_{w}{ }^{0}}\right\}^{1 / 2}\right]
\end{aligned}
$$

Here, $v_{\mathrm{p}}\left(\equiv \sum_{x} v_{x}\right)$ is the polymer volume fraction, and $X_{w}{ }^{0}$, the weight-average degree of polymerization $X$ (o means the original polymer). We neglected the concentration dependence of $\chi_{12}, \chi_{13}$, and $\chi_{23} . v_{\mathrm{p}}$ is related to $v_{1}$ and $v_{2}$ through the relation

$$
v_{1}+v_{2}+v_{\mathrm{p}}=1
$$

By solving eq 3 the spinodal curve (SC) can be directly calculated and by solving the simultaneous equations (eq 3 and 4 ) the volume fractions at CSP, $v_{1}{ }^{\mathrm{c}}, v_{2}{ }^{\mathrm{c}}$, and $v_{\mathrm{p}}{ }^{\mathrm{c}}$ are evaluated. Equation of $\mathrm{SC}$ at $v_{1}=0$ is obtained from eq 3 in the form: ${ }^{14}$

$$
v_{\mathrm{p}}+\frac{v_{2}}{X_{w}{ }^{0}}-2 \chi_{23} v_{2} v_{\mathrm{p}}=0
$$

$$
v_{1}^{(1)}=1-v_{\mathrm{p}}^{(1)}
$$

Then eq 7a and eq 9a give $v_{\mathrm{p}}{ }^{(1)}$ and $v_{\mathrm{p}}{ }^{(2)}$, separately and the co-solvency occurs only when $v_{\mathrm{p}}{ }^{(1)}$ and $v_{\mathrm{p}}{ }^{(2)}$, evaluated thus, satisfy the following two inequalities concurrently.

$$
0<v_{\mathrm{p}}^{(1)}<1 \text { and } 0<v_{\mathrm{p}}^{(2)}<1
$$

Without complete satisfaction of eq 10 the co-solvency never occur. In this sense, the reverse is rigorously true.

In other words, the necessary condition of co-solvency can also be obtained by putting eq 10. If an additional crude approximation of $X_{w}{ }^{0} \rightarrow \infty$ is applied to eq 10 the necessary condition reduces to the well-known inequalities, $X_{w}{ }^{0}$ value into eq $7 \mathrm{a}$ and $9 \mathrm{a}$, combined with 


$$
\chi_{13}>0.5 \text { and } \chi_{23}>0.5
$$

Moreover, when two CSP are obtained from eq 3 and 4 the system has two two-phase equilibria regions and unavoidably co-solvency occurs. Then, the existence of two CSP is the sufficient condition. We denote the compositions of a CSP at solvent 1 side by $v_{1}{ }^{\mathrm{c}(1)}, v_{2}{ }^{\mathrm{c}(1)}$, and $v_{\mathrm{p}}^{\mathrm{c}(1)}$ and those of another CSP at solvent 2 side by $v_{1}{ }^{\mathrm{c}(2)}, v_{2}^{\mathrm{c}(2)}$, and $v_{\mathrm{p}}^{\mathrm{c}(2)}$. When the system has larger $\chi_{12}$, smaller $\chi_{13}$ and smaller $\chi_{23}, v_{1}{ }^{\mathrm{c}(1)}$ increases and $v_{1}{ }^{\mathrm{c}(2)}$ decreases (i.e., $v_{2}{ }^{\mathrm{c}(1)}$ decreases and $v_{2}{ }^{\mathrm{c}(2)}$ increases) and the interval between two CSPs has a tendency to expand and the region of cosolvency widens significantly.

\section{COMPUTER EXPERIMENT}

SC and CSP were calculated according to the method of Kamide and Matsuda (eq 18 and 19 of ref 14). The cloud point curve (CPC) was indirectly estimated from the binodal curve (see Figures 1 and 2 of ref 14).

The calculations of SC, CSP and CPC for the quasi-ternary systems consisting of multicomponent polymers with the SchulzZimm (SZ) type molecular weight distribu-
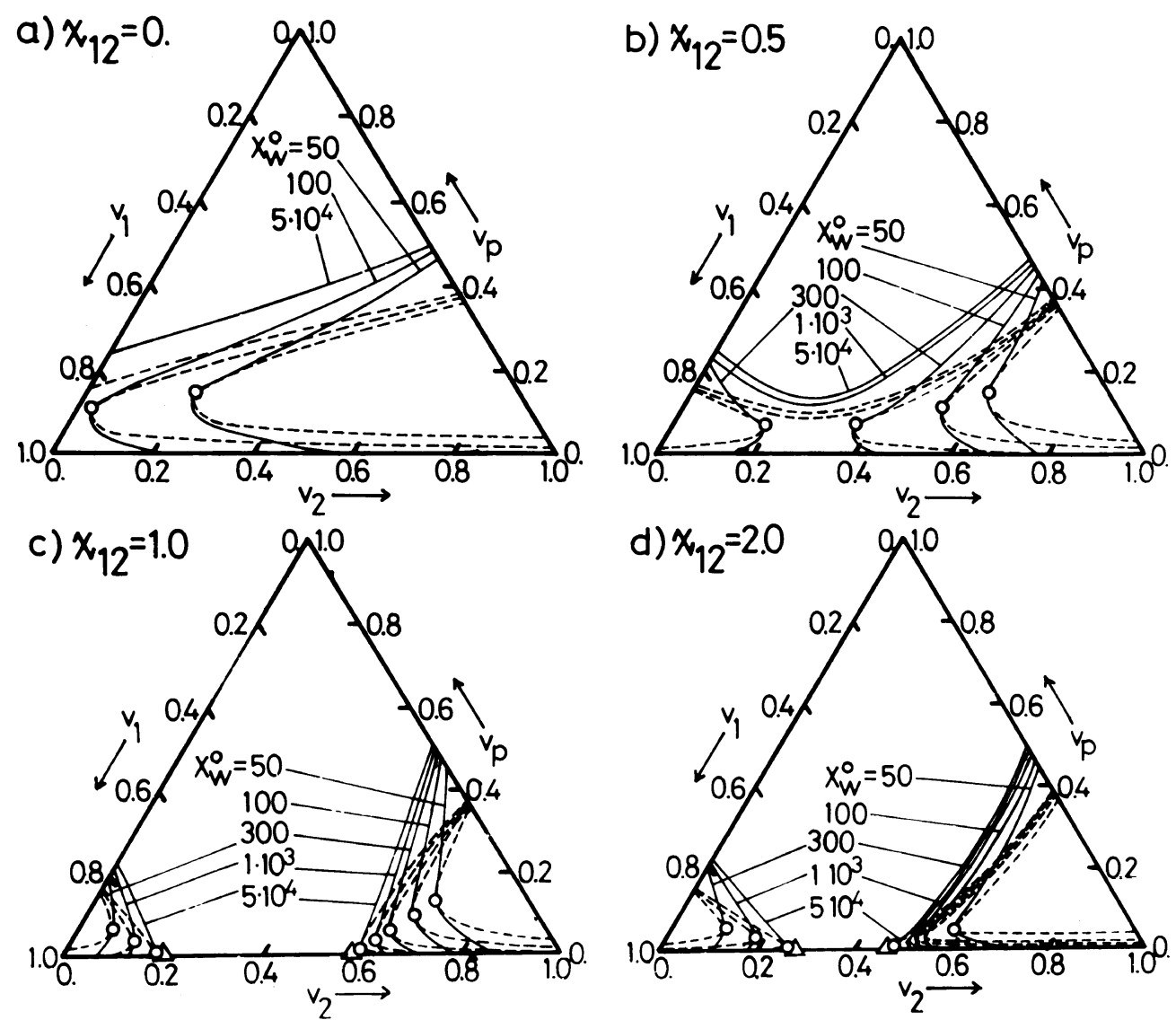

Figure 1. Effect of the weight-average degree of polymerization of the original polymer $X_{w}{ }^{0}$ on the cosolvency of quasi-ternary solution systems consisting of the multicomponent polymer (Schulz-Zimm distribution, $X_{w}{ }^{0} / X_{n}^{0}=2$ ) dissolved in a binary mixture: a) $\chi_{12}=0, \chi_{13}=0.6, \chi_{23}=0.8$; b) $\chi_{12}=0.5, \chi_{13}=0.6$, $\chi_{23}=0.8$; c) $\chi_{12}=1.0, \chi_{13}=0.6, \chi_{23}=0.8$; d) $\chi_{12}=2.0, \chi_{13}=0.6, \chi_{23}=0.8$; full line, cloud point curve; broken line, spinodal curve; unfilled circle, critical solution point; unfilled triangle, Flory solvent. 
tion $\left(X_{w}{ }^{0}=50 \sim 5 \times 10^{4}\right.$ and $X_{w}{ }^{0} / X_{n}{ }^{0}=1 \sim 5$ $\left(X_{n}^{0}\right.$ is the number average $\left.X\right)$ ) dissolved in binary solvent mixture were carried out in the ranges of $\chi_{12}=0 \sim 2, \chi_{13}=0 \sim 1.4$, and $\chi_{23}=$ $0 \sim 1.4$.

\section{RESULTS AND DISCUSSION}

Figure 1 illustrates the effect of $X_{w}{ }^{0}$ on CPC, $\mathrm{SC}$, and CSP. In the figure, the full and broken lines are CPC and SC, respectively and CSP is shown as an unfilled circle. In the case of $\chi_{12}=1.0$ and 2.0 with $\chi_{13}=0.6$ and $\chi_{23}=0.8$ (Figure 1c and 1d), the co-solvency occurs over an entire range of $X_{w}{ }^{0}$ and the systems have two Flory solvent compositions: ${ }^{27}$ The volume fraction of solvent 1 at the Flory solvent, $v_{1}{ }^{\mathrm{F}}=0.4248$ and 0.8024 for $\chi_{12}=1.0$ and $v_{1}{ }^{\mathrm{F}}$ $=0.5281$ and 0.7283 for $\chi_{12}=2.0$ (for these cases, $\chi_{13}=0.6$ and $\chi_{23}=0.8$ ). At limit of $X_{w}{ }^{0}=\infty$ two CSP coincide with their Flory solvent compositions. In the case of $\chi_{12}=0.5$, the Flory solvent composition does not exist, but in the range of relatively smaller $X_{w}{ }^{0}$ $(=300)$ two CSPs are observed and consequently the co-solvency occurs. Two twophase equilibrium regions overlap heavily with each other for polymer solutions with larger $X_{w}{ }^{0}$.

In the case of $\chi_{12}=0$ (Figure 1a), solvent 1 having $\chi_{13}=0.6$ behaves as good solvent for smaller $X_{w}{ }^{0}$ (for example, 50 and 100). For larger $X_{w}{ }^{0}$ solvent 1 with $\chi_{13}=0.6$ becomes of course poorer and no co-solvency occurs.

Even in the case when two two-phase equilibrium regions overlap as demonstrated in Figure 1a $\left(X_{w}{ }^{0}=5 \times 10^{4}\right)$ and $1 \mathrm{~b}\left(X_{w}{ }^{0}=1 \times 10^{3}\right.$ and $5 \times 10^{4}$ ), one phase region exists at higher $v_{\mathrm{p}}$. This region can also be, in a broad sense, regarded as that of co-solvent if the region maintains the liquid phase. But, in this article, we deal only with the case when two two-phase equilibrium regions are observed. Tompa ${ }^{15}$ predicted that for $\chi_{12}=2.0$ solvents 1 and 2 become critical consolute state and for $\chi_{12}>$ 2.0 three-phase separation region exists. A

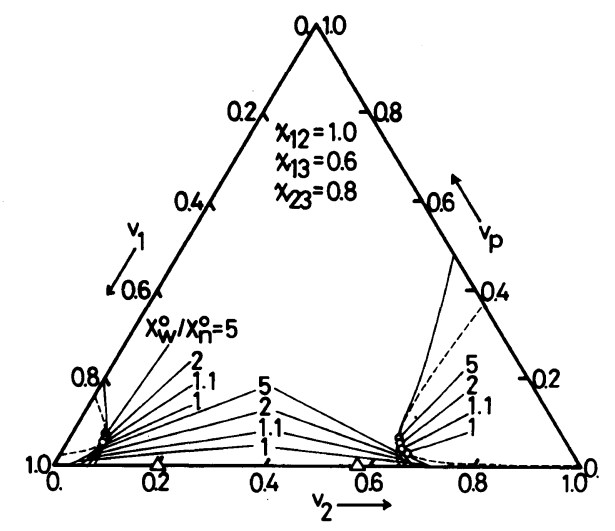

Figure 2. Effect of the ratio of the weight- to the number-average degree of polymerization of the original polymer $X_{w}{ }^{0} / X_{n}{ }^{0}$ on the co-solvency of quasi-ternary solution sytems consisting of multicomponent polymer (Schulz-Zimm distribution, $X_{w}{ }^{0}=300$ ) in a binary solvent mixture: $\chi_{12}=1.0, \chi_{13}=0.6, \chi_{23}=0.8$; full line, cloud point curve; broken line, spinodal curve; unfilled circle, critical solution point; unfilled triangle, Flory solvent.

sign of the occurrence of three phase separation can be observed at $\chi_{12}=2.0$. In Figure $1 \mathrm{~d}\left(\chi_{12}=2.0\right)$ CSP exists on CPC at solvent 2 side for $X_{w}{ }^{0}=50$ and $5 \times 10^{4}$, but no CSP exists on CPC at solvent 2 side for $X_{w}{ }^{0}=100$, 300 and $1 \times 10^{3}$. This singular property is considered to be closely correlated with threephase separation phenomena and will be discussed in more detail in a forthcoming paper.

Figure 2 shows the effect of the polymolecularity of the polymer $\left(\mathrm{SZ}, X_{w}{ }^{0}=300\right)$ on co-solvency for the case of $\chi_{12}=1.0, \chi_{13}=0.6$, and $\chi_{23}=0.8$. As eq 3 shows, SC (broken line) is independent of the polymolecularity. As $X_{w}{ }^{0} / X_{n}{ }^{0}$ increases, $v_{\mathrm{p}}{ }^{\mathrm{c}}$ increases in both two-phase equilibrium regions. In the region $v_{\mathrm{p}}{ }^{0}>v_{\mathrm{p}}{ }^{\mathrm{c}}\left(v_{\mathrm{p}}{ }^{0}\right.$ is the initial polymer volume fraction) $\mathrm{CPC}$ is sensitive to $X_{w}{ }^{0} / X_{n}{ }^{0}$ and two-phase equilibrium region widens with increasing $X_{w}^{0} / X_{n}^{0}$. These effects are similar to the effect of $X_{w}{ }^{0} / X_{n}{ }^{0}$ on CPC and CSP of the quasi-binary systems consisting of multicomponent polymers dissolved in a single solvent, first noticed by Kamide et al. ${ }^{28}$ Note that in the quasi-ternary systems $v_{\mathrm{p}}^{\mathrm{c}}$ is much smaller than that in the quasi-binary systems 

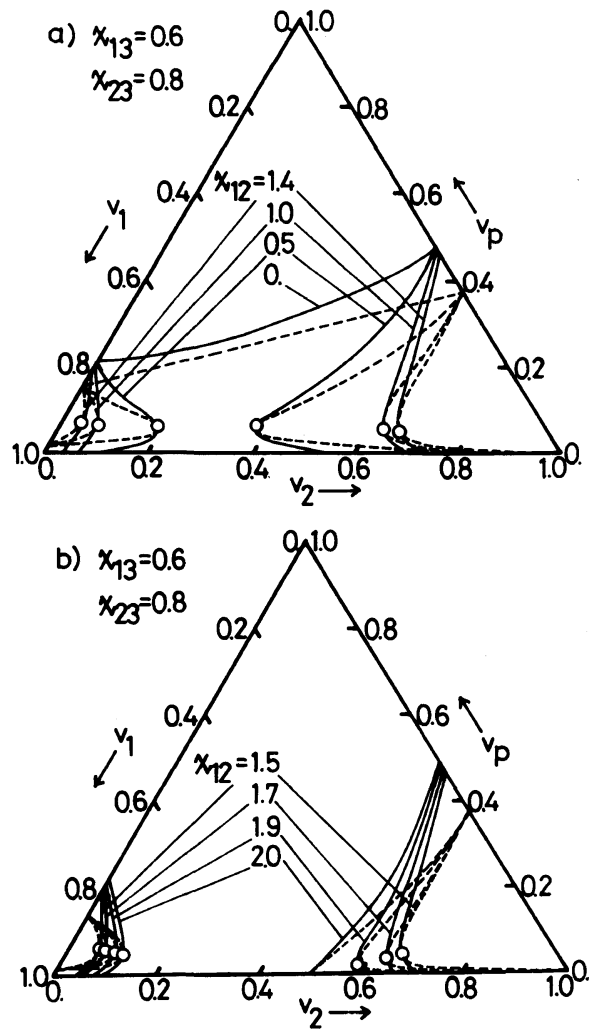

Figure 3. Effect of $\chi_{12}$ on the co-solvency of quasiternary solution systems consisting of multicomponent polymer in a binary solution: Original polymer, SchulzZimm distribution, $X_{w}{ }^{0}=300, X_{w}{ }^{0} / X_{n}{ }^{0}=2 ; \chi_{13}=0.6$ and $\chi_{23}=0.8$; full line, cloud point curve; broken line, spinodal curve; unfilled circle, critical solution point.

and the role of CPC on the total phase diagrams in the former systems is significantly small as compared with that in the latter systems.

Figure 3 shows the effect of $\chi_{12}$ on cosolvency for the quasi-ternary systems consisting of the polymer $\left(\mathrm{SZ}, X_{w}{ }^{0}=300\right)$ in a binary solvent mixture $\left(\chi_{13}=0.6\right.$ and $\chi_{23}=$ $0.8)$. In the case of $\chi_{12}=0$ no co-solvency occurs. SC at $\chi_{12}=0$ can be accurately represented by a straight line connecting the two points: $\left(0, v_{2}{ }^{(2)}, v_{\mathrm{p}}{ }^{(2)}\right)$ (SP given by eq 6) and $\left(v_{1}{ }^{(1)}, 0, v_{\mathrm{p}}{ }^{(1)}\right)$ (SP given by eq 8 ). Co-solvency occurs at $\chi_{12}=0.5$ and the region of co-solvency becomes wide as $\chi_{12}$ increases, approaching the maximum at $\chi_{12}=1.4$. Further in-

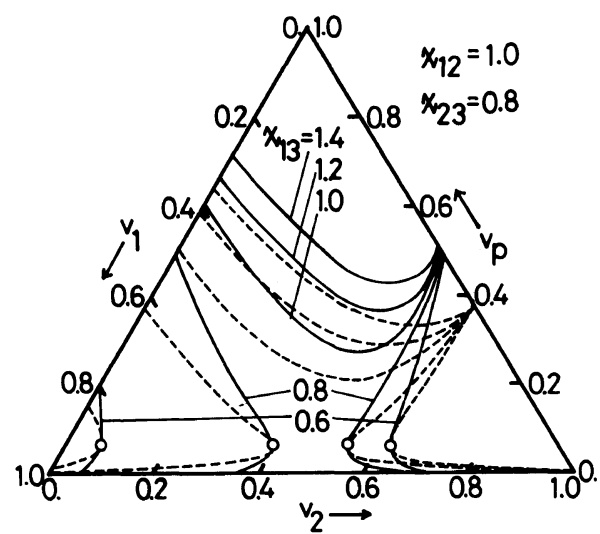

Figure 4. Effect of $\chi_{13}$ on the co-solvency of quasiternary solution systems consisting of multicomponent polymer in a binary solution: Original polymer, SchulzZimm distribution, $X_{w}{ }^{0}=300, X_{w}{ }^{0} / X_{n}^{0}=2 ; \chi_{12}=1.0$ and $\chi_{23}=0.8$; full line, cloud point curve; broken line, spinodal curve; unfilled circle, critical solution point.

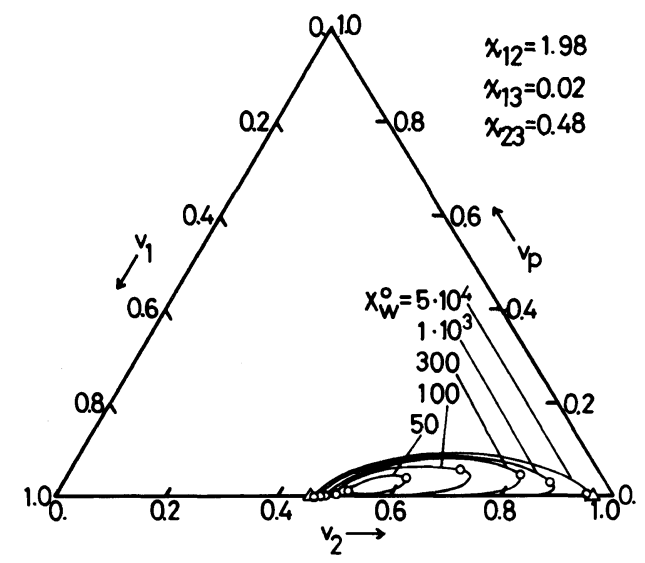

Figure 5. Effect of the weight-average degree of polymerization of the original polymer $X_{w}{ }^{0}$ on the cononsolvency of quasi-ternary solution systems consisting of multicomponent polymer (Schulz-Zimm distribution, $X_{w}{ }^{0} / X_{n}{ }^{0}=2$ ) in a binary solvent mixture: $\chi_{12}=1.98$, $\chi_{13}=0.05, \chi_{23}=0.48$; full line, spinodal curve; unfilled circle, critical solution point; unfilled triangle, Flory solvent.

crease in $\chi_{12}$ beyond 1.4 makes the co-solvency region smaller again, increasing two-phase equilibrium region. Two $v_{\mathrm{p}}^{\mathrm{c}}$ decrease slowly, but monotonously with an increase in $\chi_{12}$.

Figure 4 shows the effect of $\chi_{13}$ on cosolvency for the quasi-ternary systems consisting of the polymers $\left(\mathrm{SZ}, X_{w}{ }^{0}=300\right)$ in a 
binary solvent mixture $\left(\chi_{12}=1.0\right.$ and $\chi_{23}=$ $0.8)$. A decrease in $\chi_{13}$ brings about an expansion of a single phase region, yielding co-solvency at $\chi_{13}=0.8$ and 0.6 . At $\chi_{13}=0.8$ the system does not have two Flory solvent compositions, but at $\chi_{13}=0.6$ the system has two Flory solvent compositions $\left(v_{1}{ }^{\mathrm{F}}=0.4249\right.$ and 0.8024). ${ }^{27}$ The effect of $\chi_{23}$ on the cosolvency is principally the same as that of $\chi_{13}$.

As Tompa ${ }^{15}$ predicted as early as 1956 , the theory indicates that a mixture of solvent 1 having $\chi_{13}<0.5$ and solvent 2 having $\chi_{23}<$ 0.5 (in other words, the both solvents are good solvents when used separately) may be

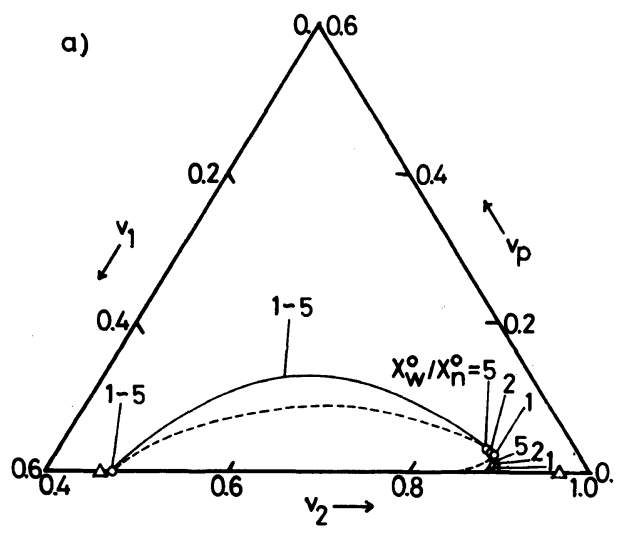

b)

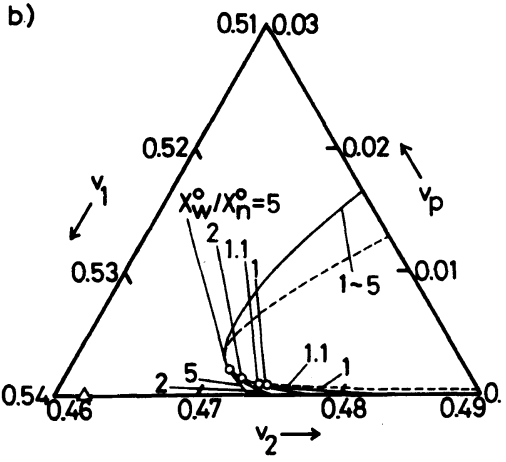

Figure 6. Effect of the ratio of the weight- to the number-average degree for polymerization of the original polymer $X_{w}{ }^{0} / X_{n}^{0}$ on the co-nonsolvency of quasiternary solution systems consisting of multicomponent polymer (Schulz-Zimm distribution, $X_{w}{ }^{0}=1000$ ) in a binary solvent mixture: $\chi_{12}=1.98, \chi_{13}=0.02, \chi_{23}=$ 0.48 ; full line, cloud point curve; broken line, spinodal curve; unfilled circle, critical solution point; unfilled triangle, Flory solvent. sometimes nonsolvent for the polymer. Unfortunately, this phenomenon has not yet been experimentally confirmed. The phenom-
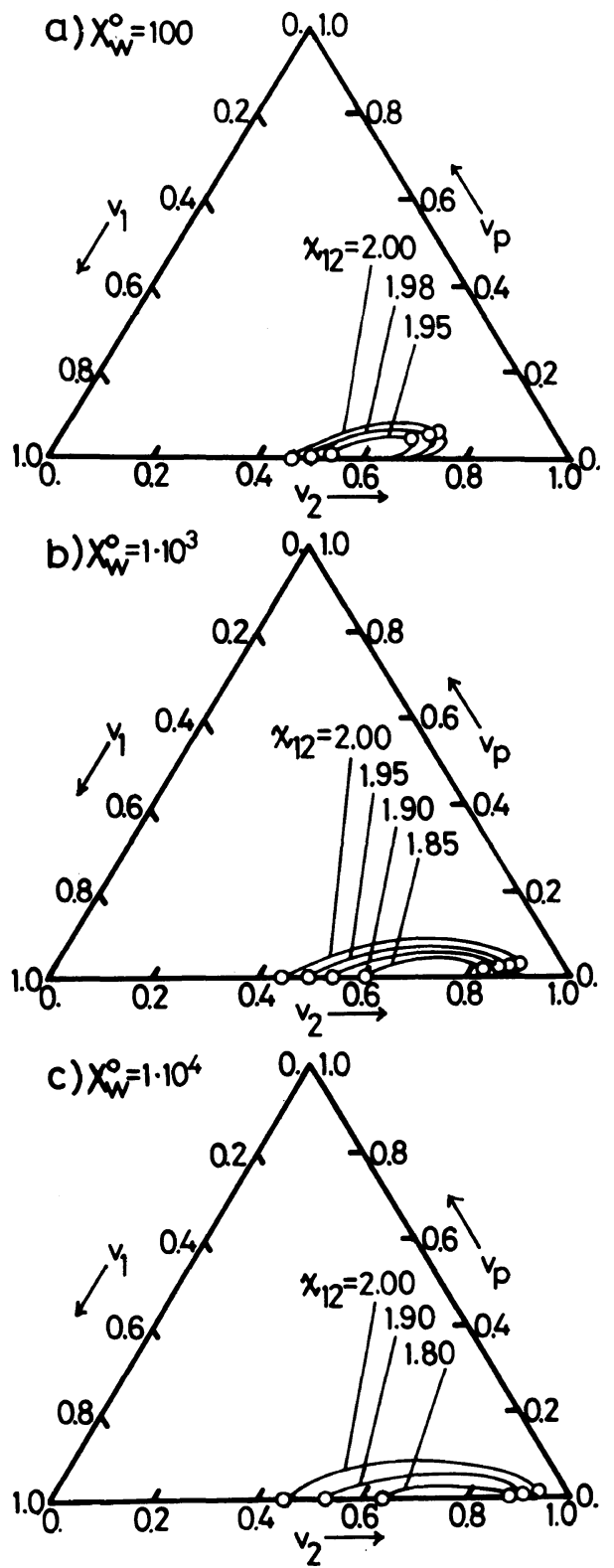

Figure 7. Effect of $\chi_{12}$ on the co-nonsolvency of quasiternary solution systems consisting of multicomponent polymer in a binary solvent mixture: Original polymer, Schulz-Zimm distribution, $X_{w}{ }^{0} / X_{n}^{0}=2, X_{w}{ }^{0}=1 \times 10^{2}$ (a), $1 \times 10^{3}(\mathrm{~b})$, and $1 \times 10^{4}$ (c); $\chi_{13}=0.02, \chi_{23}=0.48$; full line, spinodal curve; unfilled circle, critical solution point. 
enon is just reverse to co-solvency and we define this hereafter co-nonsolvency. Cononsolvency is apparently accompanied with two CSPs as co-solvency is.

Figure 5 shows the effect of $X_{w}{ }^{0}$ on cononsolvency for the quasi-ternary systems $\left(\chi_{12}=1.98, \chi_{13}=0.02\right.$, and $\left.\chi_{23}=0.48\right)$. With an increase in $X_{w}{ }^{0}$ the two-phase equilibrium region expands its space. Two CSPs, denoted as unfilled circles in the figure, reduce to two Flory solvent compositions at the limit of $X_{w}{ }^{0}=\infty$. Note that there are cases when cosolvency occurs, even if the system has no Flory solvent compositions (see, Figures $1 \mathrm{~b}$, $3 \mathrm{a}$ and 4). The system, in which co-nonsolvency occurs, has always two Flory solvent compositions.

Figure $6 \mathrm{a}$ shows the effect of the polymolecularity of the polymer $\left(\mathrm{SZ}, X_{w}{ }^{0}=1000\right)$ on co-nonsolvency of the quasi-ternary systems $\left(\chi_{12}=1.98, \chi_{13}=0.02\right.$, and $\left.\chi_{23}=0.48\right)$. The polymolecularity influences co-nonsolvency very slightly. With an increase in $X_{w}{ }^{0} / X_{n}{ }^{0}, v_{\mathrm{p}}{ }^{\mathrm{c}}$ increases significantly and in the region of $v_{\mathrm{p}}{ }^{0}<v_{\mathrm{p}}{ }^{\mathrm{c}}$ two-phase equilibria region expands very slightly with increasing $X_{w}^{0} / X_{n}^{0}$. Figure $6 \mathrm{~b}$ is an extended figure of the region enclosed by the lines $0.46<v_{2}<0.49$ and $0<v_{\mathrm{p}}<0.01$ of Figure 6a. From Figure 6a we might erroneously conclude that $\mathrm{SC}$ is intercepted with a $v_{1}-v_{2}$ axis, but Figure $6 \mathrm{~b}$ shows $\mathrm{SC}$ does not intercepted with the axis. $\mathrm{SC}$ at $v_{2}-v_{\mathrm{p}}$ axis side also has a peak and gradually approaches to $v_{1}-v_{2}$ axis with decreasing $v_{\mathrm{p}}$.

Figure 7 shows the effect of $\chi_{12}$ on cononsolvency for the quasi-ternary systems consisting of the polymers $\left(\mathrm{SZ}, X_{w}{ }^{0} / X_{n}{ }^{0}=2\right)$ in a binary mixture $\left(\chi_{13}=0.02\right.$ and $\left.\chi_{23}=0.48\right)$. With a slight increase in $\chi_{12}$ the co-solvency region increases remarkably. For larger $X_{w}^{\circ}$, this tendency is evidently accelerated and co-nonsolvency occurs at lower $\chi_{12}$. Two $v_{\mathrm{p}}^{\mathrm{c}}$ have a slight tendency to increase with $\chi_{12}$.

Figure 8 shows the effect of $\chi_{13}$ and $\chi_{23}$ on co-nonsolvency of the quasi-ternary systems consisting of the polymers (SZ, $X_{w}{ }^{0}=1 \times 10^{4}$,

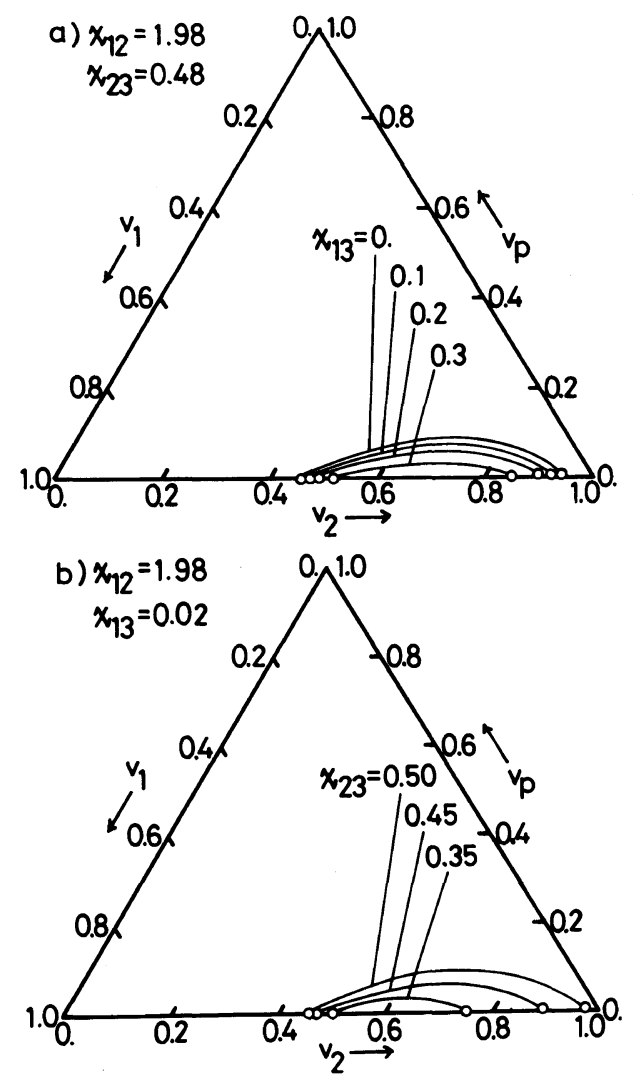

Figure 8. Effect of $\chi_{13}$ (a) and $\chi_{23}$ (b) on the cononsolvency of quasi-ternary solution systems consisting of multicomponent polymer in a binary solvent mixture: Original polymer, Schulz-Zimm distribution, $X_{w}{ }^{0}=$ $1 \times 10^{4}, X_{w}{ }^{0} / X_{n}{ }^{0}=2$; a) $\chi_{12}=1.98, \chi_{23}=0.48$; b) $\chi_{12}=$ 1.98, $\chi_{13}=0.02$; full line, spinodal curve; unfilled circle, critical solution point.

$\left.X_{w}{ }^{0} / X_{w}{ }^{0}=2\right)$ dissolved in a binary mixture. In the case of $\chi_{13}<\chi_{23}$, the co-nonsolvency region expands with decreasing $\chi_{13}$ and increasing $\chi_{23}$. With a decrease in $\chi_{13}$ co-nonsolvency region widens almost symmetrically, but the situation is somewhat different when $\chi_{23}$ increases: The expansion of solvent 2 side is much more remarkable. In the case of $\chi_{13}>\chi_{23}$ the effect of $\chi_{13}$ and $\chi_{23}$ is just reverse to that in the case of $\chi_{13}<\chi_{23}$.

\section{REFERENCES}

1. F. D. Miles, "Cellulose Nitrate," Imperial Chem. 
Industries Ltd., Oliver and Boyd, Edinburgh, London, 1955, Chapter V.

2. A. Dobry, J. Chim. Phys., 35, 387 (1938).

3. J. N. Brønsted and K. Volquvartz, Trans. Faraday Soc., 36, 619 (1940).

4. J. M. G. Cowie and J. T. McCrindle, Eur. Polym. J., 8, 1185 (1972).

5. J. M. G. Cowie and J. T. McCrindle, Eur. Polym. J., 8, 1325 (1972).

6. J. M. G. Cowie and I. J. McEven, Macromolecules, 3, 291 (1974).

7. J. M. G. Cowie and I. J. McEven, J. Chem. Soc., Faraday Trans. 1, 70, 171 (1974).

8. I. Katime, R. Valenciano, and J. M. Teijon, Eur. Polym. J., 15, 261 (1979).

9. L. Gargallo, D. Radic, and I. A. Katime, Eur. Polym. J., 17, 439 (1981).

10. I. Katime, J. R. Ochoa, and L. C. Cesteros, Eur. Polym. J., 19, 1167 (1983).

11. I. Katime and J. R. Ochoa, Eur. Polym. J., 20, 99 (1984).

12. R. L. Scott, J. Chem. Phys., 17, 268 (1949).

13. C. H. Bamford and H. Tompa, Trans. Faraday Soc., 40, 310 (1950).

14. K. Kamide and S. Matsuda, Polym. J., 18, 347 (1986).

15. H. Tompa, "Polymer Solutions," Butterwords Scientific Publishers, London, 1956, Chapter 7.
16. A. Dondos and D. Patterson, J. Polym. Sci., A-2, 7, 209 (1969).

17. P. Munk, M. T. Abizaoude, and M. E. Halbrook, J. Polym. Sci., Polym. Phys. Ed., 16, 105 (1978).

18. M. G. Prolongo, R. M. Masegosa, I. HernandezFeuntes, and A. Horta, Macromolecules, 14, 1526 (1981).

19. A. Horta and I. Fernandez-Pierola, Macromolecules, 14, 1519 (1981).

20. R. M. Masegosa, M. G. Prolongo, I. HernandezFeures, and A. Horta, Macromolecules, 17, 1181 (1984).

21. M. G. Prolongo, R. M. Masagosa, I. HernandezFeures, and A. Horta, Polymer, 25, 1307 (1984).

22. B. E. Read, Trans. Faraday Soc., 56, 382 (1960).

23. W. H. Stockmayer and M. Fixman, J. Polym. Sci., C, 1, 137 (1963).

24. M. Nakata and N. Numasawa, Macromolecules, 18 , 1736 (1985).

25. D. M. Koenhen and C. A. Smolders, J. Appl. Polym. Sci., 19, 1163 (1975).

26. K. Kamide and S. Matsuda, and Y. Miyazaki, Polym. J., 16, 479 (1984).

27. S. Matsuda and K. Kamide, Polym. J., 19, 203 (1987).

28. K. Kamide, S. Matsuda, T. Dobashi, and $M$. Kaneko, Polym. J., 16, 839 (1984). 